\title{
Análisis de las bibliografías de las asignaturas de pedagogía y didáctica de los grados en ciencias de la actividad física y el deporte en España \\ Analysis of the bibliographies of the subjects on pedagogy and didactics of physical activity and sport sciences degrees in Spain \\ Carlos Gutiérrez-García, María del Pilar Vega-Álvarez, María Teresa Gómez-Alonso, Ángel Pérez-Pueyo \\ Universidad de León (España)
}

Resumen. En este trabajo se analizan las bibliografías de las asignaturas relacionadas con la pedagogía y la didáctica presentes en los actuales grados en ciencias de la actividad física y del deporte que se imparten en las universidades españolas. Se diseñó un estudio de tipo cuantitativo y descriptivo, centrado en los ámbitos de la bibliometría relativos a productividad. La muestra final consistió en 70 bibliografías, de las que se recogieron mediante consulta directa un total de 1.365 referencias. Las variables analizadas fueron: años de publicación, títulos, autores, tipología documental y lenguas de publicación. Los resultados muestran que las obras referidas fueron publicadas entre 1966 y 2013, se concentran en las tres últimas décadas y tienen una presencia creciente hasta la primera década del s. XXI. Existe una gran diversidad en cuanto a obras y autores citados ( $n=725$ y 673 respectivamente), así como una predominancia del libro como tipología documental y del castellano como lengua de publicación (73.5 y $89.5 \%$ del total de obras respectivamente). El estudio concluye que actualmente no es posible identificar una o varias obras que sean referidas en la generalidad de estas guías. Asimismo, la posible exclusión de referencias de interés para la formación de titulados, derivada de la primacía del formato libro y del castellano sobre otros formatos e idiomas como el artículo y el inglés.

Palabras clave. Pedagogía de la actividad física, didáctica de la actividad física, grado en ciencias de la actividad física y el deporte, guías docentes, bibliografías, bibliometría.

Abstract. In this paper we analyze the bibliographies of the subjects related to pedagogy and didactics present in Spanish current Physical Activity and Sport Sciences degrees. A quantitative and descriptive study was designed, focused on the fields of bibliometrics concerning productivity. The final sample consisted of 70 bibliographies, from which a total of 1,365 references were collected by direct consultation. The variables analyzed were: years of publication, titles, authors, document types and languages of publication. The results show that the references cited in the bibliographies were published between 1966 and 2013, they are concentrated in the last three decades and have a growing presence until the first decade of the 21 ${ }^{\text {st }}$ Century. There is great diversity in terms of works and authors cited ( $n=725$ and 673 respectively), a predominance of books as document type and Castilian as language of publication (73.5 and $89.5 \%$ respectively of total works). The study concludes that it is not possible to identify one or more references which are cited in the majority of the analyzed bibliographies. Also, it points to the potential exclusion of relevant works for graduates' education, derived from the primacy of books and Castilian language with respect to other reference types such as papers and languages such us English.

Keyword. Pedagogy of physical activity, didactics of physical activity, physical activity and sports sciences degree, teaching guides, bibliographies, bibliometrics.

\section{Introducción}

La pedagogía y la didáctica han sido tradicionalmente un pilar fundamental en los estudios superiores ligados a las ciencias de la actividad física y del deporte (CCAFD) en España (Pastor, 1997, 2000). Esto se debe a que uno de los principales objetivos (si no el principal) de todas las instituciones y estudios que antecedieron a los actuales grados en CCAFD fue el de formar profesorado de educación física escolar que educase corporal y motrizmente a niños y jóvenes. De hecho, la enseñanza de la educación física en la etapa de educación secundaria ha sido desde hace décadas la principal ocupación laboral de los licenciados/ graduados en CCAFD (ANECA, 2005; González \& Contreras, 2003), y sigue estando entre las principales preferencias laborales de los futuros egresados (García, Pires, \& Fernandez, 2013). Aunque desde hace años ya se viene advirtiendo de la saturación laboral de este sector (González \& Contreras, 2003), muchas otras salidas laborales (e.g., iniciación deportiva, ocio y tiempo libre, actividad física adaptada) del cada vez más diversificado ámbito de la actividad física y deportiva demandan a sus profesionales una sólida formación para ejercer la enseñanza (Lavega, 2008; Lledó, Martínez, \& Huertas, 2014). Por ello, el ámbito educativo sigue estando muy presente en los actuales planes de estudio del grado en CCAFD (ANECA, 2005; Costes, Fuster, Castellar, \& Planas, 2014).

Como muchas otras áreas de conocimiento, la pedagogía y la didáctica de la actividad física y el deporte han sufrido constantes procesos de revisión y renovación (Fraile, 2004b; Kirk, Macdonald, \& O’Sullivan, 2006). Estos procesos han sido particularmente intensos durante las últimas décadas, al calor del propio desarrollo del conjunto de las CCAFD, generando una notable producción científica y técnica (Devís-

Fecha recepción: 09-02-16. Fecha de aceptación: 17-05-16 Carlos Gutiérrez-García

cgutg@unileon.es
Devís, Valenciano, Villamón, \& Pérez, 2010; Valenciano, Devís-Devís, Villamón, \& Peiró, 2010). Si en el ámbito europeo y norteamericano el despegue de las CCAFD se produjo a partir de mediados de la década de 1960 (Birrell, 2006), en España esto sucedió bastante tiempo después, a finales de la década de 1980 (Pastor, 2003). Así, en poco tiempo esta «exagerada aceleración histórica» (Pastor, 2004, p.15) ha creado un importante cuerpo de conocimientos en el que se han ido amalgamando formas más clásicas de conceptualizar y entender la enseñanza de la actividad física y el deporte y corrientes renovadoras provenientes de otros países y de nuestro propio contexto. Todo ello ha dado como resultado un panorama que se revela complejo, diversificado y poco uniforme.

En relación a este desarrollo, la formación pedagógica y didáctica de los titulados superiores en CCAFD ha sido estudiada en el contexto más amplio de los modelos de formación del profesorado de educación física y de los planes de estudio de las instituciones que se han encargado de la formación de dichos titulados (véanse e.g., Pastor, 1997, 2000). Desde una perspectiva crítica, Fraile (2004a) denunció, en las bases del modelo formativo actual, la desaparición de la pedagogía como conocimiento disciplinar básico en el Libro Blanco: Título de Grado en Ciencias de la Actividad Física y del Deporte (ANECA, 2005), permaneciendo solamente la didáctica como conocimiento aplicado. La formación pedagógica y didáctica también se ha estudiado desde las opiniones de profesores, alumnos y/o egresados sobre las competencias docentes relacionadas con estos estudios (véanse e.g., Gutiérrez-García, PérezPueyo, Pérez-Gutiérrez, \& Palacios-Picos, 2011; Romero, Zagalaz, Romero, \& Martínez, 2011).

No obstante, hasta donde llega nuestro conocimiento, no existe ningún estudio que analice este tema desde las fuentes bibliográficas que sirven de base durante la formación inicial de los titulados. Sobre esta base, el objetivo del presente estudio fue analizar las bibliografías de las asignaturas relacionadas con la pedagogía y la didáctica, presentes en los actuales grados en CCAFD que se imparten en las universidades espa- 
ñolas, tomando como referencia las guías docentes correspondientes al curso académico 2013-2014. Este estudio puede ser interesante al menos para: (1) Conocer cuál es la bibliografía que sustenta actualmente la formación pedagógica y didáctica de los graduados en CCAFD, y definir un punto inicial para posteriores estudios sobre su permanencia y renovación, (2) Evaluar su diversidad o uniformidad, (3) Detectar qué autores y obras pueden considerarse de referencia en este ámbito. Asimismo, y en un segundo término, también podría servir como orientación para aquellas personas interesadas o directamente implicadas en la enseñanza de la pedagogía y la didáctica de la actividad física y el deporte.

\section{Material y método}

Se realizó un estudio bibliométrico de tipo cuantitativo y descriptivo, centrado en los ámbitos relativos a productividad, a través de la revisión directa de los planes de estudio y de las guías docentes de las asignaturas de didáctica y pedagogía presentes en los grados de CCAFD de todas las universidades españolas donde estos se ofertan. Si bien los usos actuales de la bibliometría se enfocan mayoritariamente hacía la evaluación del conocimiento científico, sus aplicaciones pueden ser más amplias y revelarse como útiles para otros propósitos (véanse, e.g., Pérez-Gutiérrez \& Gutiérrez-García, 2012; Pérez-Gutiérrez, GutiérrezGarcía, \& Álvarez, 2013), como es el caso de las guías docentes.

\section{Población ymuestra}

Para cuantificar la población objeto de estudio, se localizaron todas las universidades españolas en las que se impartió el grado en CCAFD durante el curso 2013-2014, obteniendo como resultado 34 universidades (25 públicas y 9 privadas). Seguidamente se consultaron los planes de estudio de estas titulaciones y se listaron las asignaturas de pedagogía, didáctica o similares denominaciones, de carácter básico u obligatorio, que se impartían en dichos grados. El total de asignaturas listadas ascendió a 80. En un tercer paso, se recuperaron las guías docentes de dichas asignaturas para analizar la sección de bibliografía presente en las mismas. Finalmente, el número total de bibliografías analizadas ascendió a 70, ya que en la guía docente de nueve asignaturas no figuraba ninguna bibliografía y, en un caso, aún no se había publicado la guía docente por estar la titulación en fase de implantación en cursos anteriores.

\section{Variables}

A partir de las bibliografías recuperadas, las variables analizadas fueron:

1. Años de publicación. Se tomó el año de publicación citado en la bibliografía, independientemente de que pudieran existir ediciones previas en años anteriores.

2. Títulos. Se consideró el título completo de la referencia, prestando especial atención a no unir títulos que pudiesen estar duplicados pero corresponder a referencias diferentes.

3. Autores. Se registraron todos los autores, independientemente de su posición de cita en la referencia. En el caso de libros coordinados únicamente se registró al coordinador o coordinadores de la publicación si la referencia hacía alusión a la obra en su conjunto, o al autor o autores de una sección si la referencia hacía alusiónúnicamente a dicha sección.

4. Tipología documental. Se consideró una tipología documental básica (libros, libros coordinados, capítulos de libros, artículos, etc.). No se tuvieron en cuenta referencias legales ni tampoco referencias inespecíficas (por ejemplo una revista en general, sin referir artículos concretos).

5. Lenguas de publicación. Se registróla lengua de publicación de la obra citada en la bibliografía, independientemente de que esta pudiese ser una traducción de una edición original previa en otro idioma.

\section{Instrumentos de recogida de información}

Para la recogida de información se diseñaron dos hojas de cálculo en el programa Microsoft Excel (v. 2007). En la primera, denominada
«Asignaturas», se registró el nombre de la universidad, su carácter público o privado, nombre de las asignaturas relacionadas con la pedagogía y con la didáctica presentes en cada plan de estudios, curso correspondiente a las mismas, su carácter básico u obligatorio, número total de créditos, el enlace a la guía docente de la asignatura, y un último campo de observaciones (por ejemplo para hacer constar si la guía docente de la asignatura no contenía bibliografía).

En la segunda hoja de cálculo, denominada «Bibliografía», se registraron individualmente todas las referencias extraídas de las 70 bibliografías analizadas, relacionando las mismas con las universidades y asignaturas correspondientes. Se añadieron campos específicos en relación a las variables analizadas (autores, títulos, años, idiomas y tipología documental).

\section{Procedimiento de recogida de información}

La recogida de datos se realizó a través de las fuentes primarias, esto es, las guías docentes de las asignaturas objeto de estudio. Las referencias bibliográficas de cada una de las guías docentes fueron trasladadas individualmente a la hoja de cálculo «Bibliografía», completando posteriormente el resto de campos de cada referencia (lengua de publicación, idioma, tipología documental, etc.).

Debido a las carencias e importantes errores que presentaban un gran número de bibliografías respecto a los elementos formales de cada una de las referencias (autores, años, títulos, etc.), fue necesario depurar y normalizar dichas referencias. También fue necesario realizar hasta cuatro rondas de verificación, independientes e intercaladas, por parte de los miembros del equipo investigador, con el objeto de lograr una base de datos final fiable sobre la que realizar el análisis. Los procesos descritos en este párrafo fueron los que necesitaron más tiempo en el conjunto de la investigación.

\section{Análisis y presentación de los datos}

Los datos obtenidos fueron analizados mediante estadística descriptiva (frecuencias y porcentajes) mediante el paquete estadístico SPSS (v. 19). La mayor parte de resultados obtenidos se presentan en tablas para su mejor comprensión.

\section{Resultados}

El número total de referencias registradas en la base de datos ascendió a 1365. Las guías docentes analizadas presentaron una gran disparidad en el número de referencias citadas, oscilando entre 2 y 112.

\section{Años de publicación}

Con respecto a la distribución temporal de las referencias, esta comprende prácticamente medio siglo, situándose en el intervalo 19662013. Como muestra la Figura 1, las referencias hasta la primera mitad de la década de 1980 son escasas, incrementándose desde este momento hasta la primera mitad de la primera década del siglo XXI, para descender seguidamente hasta la actualidad.

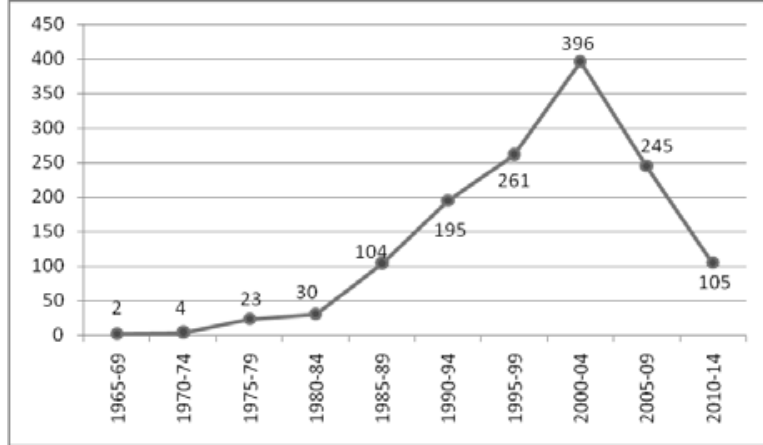

Figura 1

Distribución temporal de la bibliografía de las asignaturas de pedagogía y la didáctica de los grados en CCAFD españoles 


\section{Títulos}

El total de títulos referidos en los programas de las asignaturas ascendió a 725. La Tabla 1 muestra las obras que acumulan siete o más citas. Un total de nueve obras son citadas en seis guías docentes (8.6\%), ocho obras en cinco (7.1\%), diez obras en cuatro (5,7\%), y 665 obras estás presentes en una a tres guías docentes (1.4 a 4.3\%).

Tabla 1

Títulos más citados en las bibliografías de las asignaturas de pedagogía y la didáctica de los grados en CCAFD españoles \begin{tabular}{lcc}
\hline \multicolumn{1}{c}{ Obras (Autores, años) } & $\boldsymbol{n}$ & $\mathbf{\%}$ \\
\hline Aprender a enseñar la educación física (D. Siedentop, 1998, 2008) & 24 & 34,3 \\
Didáctica de la educación física: un enfoque constructivista (O. R. Contreras, 1998, & 22 & 31,4
\end{tabular} 2004, 2009)

Educación física y estilos de enseñanza (A. Sicilia y M. A. Delgado, 2002) $22 \quad 31,4$

Enseñar por competencias en educación física (D. Blázquez y E. M. Sebastiani, $21 \quad 30,0$

2009, 2010)

La enseñanza de la educación física. La reforma y los estilos de enseñanza (M. 19 27,1

Mosston y S. Ashworth, 1993, 1996, 1999, 2001)

Bases para una didáctica de la educación física y el deporte (F. Sánchez, 1984, 18 25,7

1986, 1989, 1990, 1992)

Para una enseñanza eficaz de las actividades físico-deportivas (M. Pierón, 1999, 18 25,7

2005)

Bases educativas de la actividad física y el deporte (B. Vázquez, 2001) $\quad 17 \quad 24,3$

Didáctica de la educación física: una perspectiva crítica y transversal (A. Fraile, 17 24,3

2004)

Planificar en educación física (J. Viciana, 2002)

Evaluar en educación física (D. Blázquez, 1990, 1992, 2008, 2009) $\quad \begin{array}{rr}17 & 24,3 \\ 16 & 22,9\end{array}$

Didáctica de las actividades física y deportivas (M. Pieron, 1988) 1420,0

Enseñar educación física en secundaría: motivación, organización y control (J. 14 20,0

Florence, 2000)

Educación física, movimiento y currículum (P. J. Arnold, 1990, 1991) $12 \quad 17,1$

$\begin{array}{lrl}\text { La lección de educación física (P. Seners, 2001) } & 12 & 17,1 \\ \text { Manual de didáctica de la educación física I. Una perspectiva constructivista } & 12 & 17,1\end{array}$

integradora. Funciones de impartición (Antonio D. Galera, 2001)

La evaluación formativa como instrumento de aprendizaje en educación física (J. 11 15,7 Díaz, 2005)

Educación física y currículum (D. Kirk, 1990) $\quad 10 \quad 14,3$

Educación física, deporte y currículum: investigación y desarrollo curricular (J. 10 14,3

Devís-Devís, 1996)

Buscando alternativas a la hora de entender y practicar la educación física escolar $\quad 9 \quad 12,9$

(V. M. López, R. Monjas y D. Pérez, 2003)

Cómo se enseñan los deportes (C. Target y J. Cathelineau, 2002) 9

Evaluación de programas en ciencias de la actividad física (F. J. Castejón, 2007) $\quad 9 \quad 12,9$

Manual de didáctica de la educación física II: Una perspectiva constructivista 9

integradora. Funciones de programación (D. Galera, 2001)

$\begin{array}{llll}\text { Actitudes, valores y normas en la educación física y el deporte: reflexiones y } & 8 & 11,4 \\ \text { propuestas didácticas (M. Prat, M. Prat y S. Soler, 2003) }\end{array}$

Cómo programar en educación física paso a paso (S. del Valle y M. J. García, 2007) $8 \quad 11,4$

Lo enseñanza de la educación física: análisis de las primeras experiencias docentes $8 \quad 11,4$

\begin{tabular}{l} 
(L. A. Ramos \& F. del Villar, 2005) \\
La nalisis de las primeras experiencias docentes $8 \quad 11,4$ \\
\hline
\end{tabular}

La otra cara de la enseñanza. La educación física desde una perspectiva crítica (A. 8
Sicilia y J. M. Fernández-Balboa, 2005)

$\begin{aligned} & \text { Nuevas perspectivas curriculares en educación física: la salud y los juegos } \\ & \text { modificados (J. Devís-Devís y C. Peiró, 1992) }\end{aligned}$
11,4

$\begin{array}{lll}\text { Educación fisica: la escuela y sus profesores (R. Tinning, 1992) } & 7 & 10,0\end{array}$

$\begin{array}{llll}\begin{array}{l}\text { La educación física en secundaria basada en competencias. Proyecto curricular y } \\ \text { programación (F. Urueña, 2010) }\end{array} & 10,0\end{array}$

La evaluación en educación fisica: investigación y práctica en el ámbito escolar (J. $7 \quad \begin{array}{lll}7 & 10,0\end{array}$

$\begin{array}{llll}\text { La iniciación deportiva y el deporte escolar (D. Blázquez, 1995, 1999) } & 7 & 10,0\end{array}$

Los estilos de enseñanza en educación física. Propuesta para una reforma de la $7 \begin{array}{lll}7 & 10,0\end{array}$

enseñanza (M. A. Delgado, 1991)

Nota: Porcentaje respecto al total de guías docentes analizadas $(n=70)$. Solo se han registrado las
ediciones citadas en las bibliografías de las asignaturas.

Tabla 2

Autores más citados en las bibliografías de las asignaturas de pedagogía y la didáctica de los grados en CCAFD españoles

Autores

Maurice Pierón

Miguel Angel Delgado

Álvaro Sicilia

Onofre Ricardo Contreras

Fernando Sánchez Bañuelos

Enric Maria Sebastiani

José Devís-Devís

Víctor Manuel López Pastor

Daryl Siedentop

Muska Mosston

Francisco Javier Castejón

Antonio Fraile

Benilde Vázquez

Jesús Viciana

Juan Luis Hernández Álvarez

Antonio D. Galera

Luis Miguel Ruiz Pérez

Sara Ashworth

Jordi Díaz Lucea

Roberto Velázque

Jacques Florence

Juan Miguel Fernández-Balboa

Fernando Ureña

Fernando del Villar

Nota: $\%$ citas = porcentaje respecto al total de entradas de autores registradas en la base de

datos $(n=1923)$; $\%$ guías = porcentaje respecto al total de de guías docentes analizadas $(n=$ datos.

\section{Autores}

Se contabilizaron un total de 1923 entradas, que correspondieron a 673 autores. La Tabla 2 muestra los 25 autores más citados. Un total de 446 autores son citados una única vez (66.3\% del total de autores y $23.2 \%$ del total de entradas), 93 dos veces (13.8\% del total de autores y 9.7\% del total de entradas), 42 tres veces (6.2\% del total de autores y $6.5 \%$ del total de entradas), y 67 entre 4 y 15 veces (10.0\% del total de autores y $25.7 \%$ del total de entradas).

\section{Tipología documental}

Respecto a la tipología documental, la Tabla 3 muestra una gran predominancia del libro sobre otras tipologías documentales. Así, los libros, libros coordinados y capítulos de libro suponen en conjunto un 88.1\% del total de referencias citadas.

Tabla 3

Tipologías documentales de la bibliografía de las asignaturas de pedagogía y la didáctica de los grados en CCAFD españoles

Tipologías documentales

Libro

Artículo

Libro coordinado

Capítulo de libro

Actas

Recurso electrónico

Tesis

Nota: Porcentaje respecto al número total de referencias citadas $(\mathrm{n}=725)$.

Tabla 4

Idiomas de la bibliografía de las asignaturas de pedagogía y la didáctica de los grados en CCAFD españoles

\begin{tabular}{lll} 
en CCAFD españoles & $\mathbf{n}$ & $\mathbf{\%}$ \\
\hline Idiomas & 649 & 89.5 \\
\hline Castellano & 51 & 6.9 \\
Inglés & 15 & 2.1 \\
Catalán & 6 & 0.8 \\
Portugués & 1 & 0.1 \\
Gallego & 3 & 0.4 \\
Francés & 3 &
\end{tabular}

Nota: Porcentaje respecto al número total de referencias citadas $(n=725)$

\section{Idiomas}

Por último, respecto a los idiomas se registraron un total de seis. El castellano destaca mucho respecto al resto de idiomas, acumulando prácticamente el 90\% del total de referencias citadas (Tabla 4).

\section{Discusión}

Los resultados obtenidos en este trabajo muestran que la mayor parte de referencias citadas en las bibliografías de las asignaturas relacionadas con la pedagogía y la didáctica de los actuales grados en CCAFD españoles fueron publicadas en las tres últimas décadas. En conjunto, estas bibliografías son diversas en cuanto a autores y títulos, mientras que existe una mayor uniformidad en cuanto a tipologías documentales e idiomas. Seguidamente procederemos a realizar una discusión general de estos resultados.

En primer lugar, el número de referencias publicadas en la segunda mitad de la década de 1980 presentes en las guías docentes marca un punto de inflexión positivo respecto a periodos anteriores. Esto es coincidente con los años en que Pastor (2003) sitúa el verdadero inicio de la investigación española en educación física. Durante estos años y principios de la década de 1990 se produjeron una serie de hechos relevantes para el desarrollo de las CCAFD en España, como fueron la creación de nuevos centros de formación y la incorporación definitiva de las enseñanzas de educación física a la universidad, la creación de la especialidad educación física en los estudios de magisterio, la preparación e implantación de una nueva reforma educativa, o la diversificación del campo profesional de los titulados (Pastor, 2000). Todo ello creó una situación propicia para que un colectivo relativamente nuevo y cada vez más numeroso de docentes e investigadores universitarios en el ámbito de las CCAFD, diese un fuerte impulso a este campo del conocimiento. Así, el 25\% de las obras más citadas (Tabla 1) corresponden a esta etapa e incluso al periodo anterior, y pueden considerarse con toda propiedad «clásicos» de la pedagogía y la didáctica de la actividad física y el deporte en España (Arnold, 1990; Blázquez, 1990; Devís-Devís \& Peiró, 1992; Kirk, 1990; Mosston \& Ashworth, 1993; Piéron, 1988; 
Sánchez, 1984).

Como había sucedido en épocas anteriores, la mayor renovación de la pedagogía y de la didáctica de la actividad física y del deporte desde esta segunda mitad de la década de 1980 siguió viniendo de la mano de la educación física escolar (Pastor, 2000). Según muestran nuestros resultados de títulos más citados, donde son mayoritarios aquellos que refieren específicamente a la educación física, esta tendencia continúa en la actualidad en la formación de los graduados en CCAFD. Además de la propia tradición disciplinar, otros factores explicativos de esta tendencia son los aires de renovación que trajo la reforma educativa de 1990 (véase López \& Gea, 2010, Martínez, 2016) y la producción realizada por profesorado universitario de escuelas de magisterio y/o facultades de educación, más enfocado hacia la educación física escolar que las Facultades de CCAFD. Por otra parte, es importante considerar que muchos otros ámbitos de la actividad física (e.g., deporte escolar, iniciación deportiva, actividad física y deporte adaptado y para poblaciones especiales, deporte para todos, etc.) pueden compartir en gran medida los principales objetivos de la educación física escolar, ligados al desarrollo integral de la persona y a la utilización de la actividad física y deporte para tal fin (Díaz \& Mandado, 2004; Fraile, 2004c; González, Manrique, \& López, 2012; Gutiérrez, 2004; Hoyos, Gutiérrez-García, \& Pérez-Pueyo, 2011; Valdemoros, 2010). Por ello, y a pesar de las diferencias que puedan separar estos ámbitos, parece lógico que la pedagogía y la didáctica de la educación física escolar sigan inspirando los programas de las asignaturas relacionadas con estas disciplinas en los grados de CCAFD.

Siguiendo esta línea temporal, el número de referencias publicadas desde la segunda mitad de la década de 1980 hasta la primera mitad del siglo XXI presentes en las guías docentes se incrementa notablemente. Se trata de un período en el que se consolidó la inercia anterior de desarrollo de las CCAFD con la implantación de estos estudios en nuevas universidades, aumento del número de programas específicos de doctorado, presencia de las CCAFD en planes nacionales de investigación, o de revistas científicas (Devís-Devís, Antolín, Villamón, Moreno, \& Valenciano, 2003; Ferro \& Floría, 2011). En elámbito de la educación no universitaria se produjo la plena implantación de la reforma educativa de 1990, y numerosos investigadores y grupos de trabajo sobre educación física escolar se unieron al movimiento renovador iniciado a principios de la década (López \& Gea, 2010; López, Monjas, \& Fraile, 2004). Todo ello dio como resultado un incremento de la producción científica y técnica sobre pedagogía y didáctica de la actividad física, parte de la cual ha pasado a formar parte de las guías docentes de las asignaturas analizadas.

Para terminar, el número de referencias citadas en las guías docentes, y publicadas en los dos últimos periodos analizados (2005-2009, y 2010-2014), sufre un acusado descenso respecto al periodo anterior (2000-2004), de un 38\% y un 73\% respectivamente. A pesar del mantenimiento de los factores favorecedores de la producción científica y técnica citados para la etapa anterior (por ejemplo, entre 2001 y 2012 se pasó de 15 a 31 universidades que ofrecían la licenciatura/grado en CCAFD), entendemos que han existido otros que explicarían este cambio de tendencia. Entre los mismos: (1) la necesidad de un cierto intervalo temporal para que la bibliografía más reciente se difunda y en su caso pase a formar parte de las guías docentes; (2) la cada vez mayor preeminencia del artículo científico sobre otras tipologías documentales de difusión del conocimiento como el libro (Valenciano, Devís-Devís, \& Villamón, 2008), cuando el artículo científico parece no ser tan considerado como los libros para su presencia en las guías docentes, como así muestran nuestros resultados; (3) los efectos colaterales de la evaluación de la actividad investigadora de la comunidad universitaria, que puede estar alterando prioridades y tendencias anteriores de publicación y dando lugar a una producción intelectual más preocupada del impacto científico que de la repercusión social, pedagógica y profesional de los trabajos (Devís-Devís, Villamón, \& Valenciano, 2014; Valenciano, 2012); (4) la permanencia de los postulados de la reforma educativa de 1990, a pesar de los cambios promovidos por las posteriores leyes educativas (LOCE, LOE, LOMCE), y en los que únicamente puede citarse como innovación significativa elámbito de las competencias, que síha generado una cierta cantidad de literatura referida en las guías docentes (e.g., Blázquez \& Sebastiani, 2009; Contreras \& Cuevas, 2011; Ureña, 2010); (5) los condicionantes existentes en algunas universidades, en las que se restringe el número máximo de referencias que pueden citarse en las guías docentes, resultando que el profesorado se ciñe a las referencias que considera más «clásicas» y consolidadas; y (6) el difícil contexto económico durante los últimos años en España, que ha puesto en duda la viabilidad de editar las obras académicas más habituales en las bibliografías, como los libros y manuales.

Por lo que se refiere a los títulos citados, estos destacan por su diversidad. Un 91.7\% de ellos están presentes únicamente en tres o menos de las 70 bibliografías analizadas, y el título más citado figura en algo más de un tercio de las mismas. Sobre la base de estos resultados, puede afirmarse que no existe una obra de referencia en el ámbito de la pedagogía y la didáctica de la actividad física que logre un amplio consenso, ya sea total o parcial, en relación a los contenidos propios de estas materias, para su integración en las guías docentes. Ello a pesar del carácter generalista de un buen número de títulos (véase Tabla 1), que enfocan la enseñanza de la educación física o de la actividad física desde una perspectiva global. Antes al contrario, las fuentes documentales citadas en las guías docentes parecen confirmar la gran heterogeneidad de fuentes utilizadas en las materias.

En consonancia con los resultados anteriores, el número de autores citados en las biografías de las guías docentes es muy amplio, y un 86.3\% de ellos son citados tres o menos veces. En este caso, el número de veces que un autor es citado no tienen por qué corresponder a su presencia en un número igual de guías docentes, ya que un mismo autor puede ser referido varias veces en la misma guía docente con obras diferentes. Esto sucede por ejemplo con obras de Domingo Blázquez (Blázquez, 1990; Blázquez \& Sebastiani, 2009), José Devís (DevísDevís, 1996; Devís-Devís \& Peiró, 1992), Alvaro Sicilia (Sicilia \& Delgado, 2002; Sicilia \& Fernández-Balboa, 2005) o Antonio D. Galera (Galera, 2001a, 2001b), autores de algunas de las obras destacadas en este estudio como las más citadas.

Respecto a autores, los más citados en las guías docentes son españoles, y entre los autores extranjeros se observan influencias especialmentedelámbito anglosajón(e.g., Arnold, 1990; Kirk, 1990; Mosston, 1978; Mosston \& Ashworth, 1993; Siedentop, 2008) y francófono (e.g., Florence, 1991; Florence, Brunelle, \& Carlier, 2000; Piéron, 1988, 1999; Seners, 2001). Esto no significa que las ideas y corrientes extranjeras sobre la pedagogía de la didáctica de la actividad física tengan poco peso en las bibliografías de las asignaturas, ya que muchas obras firmadas por autores españoles recogen esta influencia, como es el caso paradigmático de Devís-Devís y Peiró (1992) o Sicilia y FernándezBalboa (2005), entre otros. Las obras de autores extranjeros se citan mayoritariamente en su traducción al castellano y menos de un $10 \%$ de las referencias de las guías docentes están escritas en otras lenguas diferentes a las oficiales de España. En este sentido, parece existir una cierta barrera idiomática que puede deberse a las propias preferencias y/ o limitaciones del profesorado encargado de diseñar las guías docentes, ya que es innegable la calidad de mucha literatura sobre la temática que viene publicándose en el extranjero, particularmente en inglés, y que no ha sido traducida al castellano (e.g., Kirk, et al., 2006).

Por último, resulta interesante señalar que muchos de los autores españoles más citados ocupan puestos relevantes en el ámbito de la investigación en sus respectivas áreas de conocimiento (Didáctica de la Expresión corporal o Educación Física y Deportiva). Así, de los 20 autores españoles que de muestran en la Tabla 2, ocho figuran en el primer cuartil en sus áreas según H Index Scholar (Grupo de investigación EC3, 2012). Esto indica una posible relación entre producción científica y creación de referencias reconocidas para su utilización en la docencia universitaria, y respalda la controvertida idea de la estrecha y positiva reciprocidad que debería existir entre docencia e investigación en la universidad (Barnett, 2008; Sancho, 2001). No obstante, aquí es importante señalar, como se ha avanzado anteriormente, que el «formato libro» (libro, libro coordinado, capítulo de libro) supone práctica- 
mente el 90\% de las referencias citadas en las guías docentes, hecho que contrasta con el modelo actual de difusión y valoración del conocimiento científico, en el que se impone el artículo sobre otros formatos. Por el contrario, nuestro estudio revela el mayor impacto de los libros en las guías docentes y por extensión en la formación pedagógica y didáctica que reciben los graduados en CCAFD. Esto puede suponer una limitación en aspectos como la calidad del conocimiento que reciben los alumnos, la actualidad del conocimiento que se les imparte, o la propia visibilidad de las fuentes, aspectos todos ellos en los que las revistas científicas presentan elementos de superioridad respecto a otros medios de difusión del conocimiento (Valenciano, et al., 2008)

\section{Conclusiones}

El estudio realizado ha mostrado que la gran mayoría de las referencias presentes en las guías docentes de las asignaturas de pedagogía y didáctica de los grados en CCAFD en España han sido publicadas en las tres últimas décadas, hecho que coincide con el propio desarrollo de las CCAFD en nuestro país. Estas referencias son muy diversas en cuanto a títulos y autores y no es posible identificar una o varias obras de referencia que figuren en la generalidad de las guías docentes analizadas. Los autores más citados son en su mayoría españoles y cerca de la mitad ocupa posiciones relevantes en producción científica en sus áreas de conocimiento. Se aprecia también la influencia de algunos autores extranjeros, fundamentalmente del ámbito anglosajón y francófono. Las referencias analizadas se han publicado mayoritariamente en formato de libro y en idioma castellano, lo que podría estar excluyendo referencias de interés para la formación de los titulados.

\section{Referencias}

ANECA. (2005). Libro blanco: Título de grado en Ciencias de la Actividad Físicay del Deporte. Recuperado de http://www.aneca.es/ var/media/150296/libroblanco_deporte_def.pdf

Arnold, P. J. (1990). Educación física, movimiento y curriculum. Madrid: Morata.

Barnett, R. (Ed.). (2008). Para una transformación de la universidad: Nuevas relaciones entre investigación, saber y docencia. Barcelona: Octaedro.

Birrell, S. (2006). Sport and Sport Studies. En C. Rojek, S. Shaw \& T. Veal (Eds.), The Handbook of Leisure Studies (pp. 335-353). London: Palgrave Macmillan.

Blázquez, D. (1990). Evaluar en educación física. Barcelona: Inde.

Blázquez, D., \& Sebastiani, E. M. (2009). Enseñar por competencias en educación física. Barcelona: Inde.

Contreras, O. R., \& Cuevas, R. (Eds.). (2011). Las competencias básicas desde la educación física. Barcelona: Inde.

Costes, A., Fuster, J., Castellar, C., \& Planas, A. (2014). Las competencias específicas en las webs de los grados universitarios de Ciencias de la Actividad Física y Deporte. Retos: nuevas tendencias en educación física, deporte y recreación, 25, 43-47. Recuperado de http://recyt.fecyt.es/index.php/retos/article/viewFile/34473/18610

Devís-Devís, J. (1996). Educación física, deporte y currículum: investigación y desarrollo curricular. Madrid: Visor.

Devís-Devís, J., Antolín, L., Villamón, M., Moreno, A., \& Valenciano, J. (2003). Las revistas científico-técnicas españolas de las ciencias de la Actividad Física y el Deporte: inventario y análisis de la calidad de contenido y difusión. Revista española de documentación científica, 26(2), 177-190. doi:10.3989/redc.2003.v26.i2.136

Devís-Devís, J., \& Peiró, C. (1992). Nuevas perspectivas curriculares en educación física: la salud y los juegos modificados. Barcelona: Inde.

Devís-Devís, J., Valenciano, J., Villamón, M., \& Pérez, V. (2010). Disciplinas y temas de estudio en las ciencias de la actividad física y el deporte. Revista Internacional de Medicina y Ciencias de la Actividad Física y del Deporte, 10(37), 150-166. Recuperado de http:// cdeporte.rediris.es/revista/revista37/artdisciplinas147.htm
Devís-Devís, J., Villamón, M., \& Valenciano, J. (2014). Revistas iberoamericanas de Educación Física/Ciencias del Deporte presentes en Web of Science: evaluación y desafíos. Revista Brasileira de Ciências do Esporte, 36(4), 723-732. Recuperado de http:// www.sciencedirect.com/science/article/pii/S0101328914000055

Díaz, P., \& Mandado, A. (2004). Deporte y educación: pautas para hacer compatible el rendimiento y el desarrollo integral de los jóvenes deportistas. Revista de educación, 335, 35-44. Recuperado de http://www.revistaeducacion.mec.es/re335/re335_05.pdf

Ferro, A., \& Floría, P. (2011). Sports Science in the Spanish National Research, Development and Innovation Plan. A historical overview. Journal of Human Sport and Exercise, 6(3), 554-572. doi:10.4100/ jhse.2011.63.09

Florence, J. (1991). Tareas significativas en educación física escolar: una metodología para la enseñanza de los ejercicios en la animación del grupo-clase. Barcelona: Inde.

Florence, J., Brunelle, J., \& Carlier, G. (2000). Enseñar educación física en secundaria: motivación, organizacióny control. Barcelona: Inde.

Fraile, A. (2004a). Reclamando la presencia de los fundamentos pedagógicos en la formación del profesorado de educación física. Revista española de educación física y deportes, 1, 71-104. Recuperado de http://revista.consejo-colef.es/index.php/RFEED_375/article/ viewFile/13/13

Fraile,A.(Ed.). (2004b). Didáctica de la educación física: una perspectiva crítica y transversal. Madrid: Biblioteca Nueva.

Fraile, A. (Ed.). (2004c). El deporte escolar en el siglo XXI: análisis y debate desde una perspectiva europea. Barcelona: Graó.

Galera,A. D. (2001a). Manual de didáctica de la educación física I: Una perspectiva constructivista integradora. Funciones de impartición. Barcelona: Paidós Ibérica.

Galera, A. D. (2001b). Manual de didáctica de la educación física II: Una perspectiva constructivista integradora. Funciones de programación Barcelona: Paidós Ibérica.

García, J., Pires, F., \& Fernandez, J. (2013). Preferencias profesionales de los estudiantes de licenciatura en Ciencias de la Actividad Física y del Deporte de la Universidad de Sevilla. Retos: nuevas tendencias en educación física, deporte y recreación, 23, 39-42. Recuperado de http://recyt.fecyt.es/index.php/retos/article/viewFile/34565/ 18689

González, J. M., \& Contreras, O. R. (2003). Evolución de las tendencias profesionales del titulado en educación física y en ciencias de la actividad física y del deporte desde 1975 hasta la actualidad. Apunts: Educación física y deportes, 73, 19-23. Recuperado de http:// www.revista-apunts.com/es/hemeroteca?article $=308$

González, M., Manrique, J. C., \& López, V. M. (2012). Valoración del primer curso de implantación de un programa municipal integral de deporte escolar. Retos: nuevas tendencias en educación física, deporte y recreación, 21, 14-18. Recuperado de http://recyt.fecyt.es/ index.php/retos/article/viewFile/34597/18717

Grupo de investigación EC3. (2012). H Index Scholar, from http:// hindexscholar.com

Gutiérrez-García, C., Pérez-Pueyo, Á., Pérez-Gutiérrez, M., \& Palacios-Picos, A. (2011). Percepciones de profesores y alumnos sobre la enseñanza, evaluación y desarrollo de competencias en estudios universitarios de formación de profesorado. Cultura y Educación, 23(4), 499-514.

Gutiérrez, M. (2004). El valor del deporte en la educación integral del ser humano. Revista de educación, 335, 105-126. doi: 10.1174/ 113564011798392451

Hoyos, L.A., Gutiérrez-García, C., \& Pérez-Pueyo, Á. (2011). Deporte escolar: fundamentación teórica. Armenia Kinesis.

Kirk, D. (1990). Educación física y currículum. Valencia: Universidad deValencia.

Kirk, D., Macdonald, D., \& O’Sullivan, M. (Eds.). (2006). Handbook of Physical Education. London: SAGE.

Lavega, P. (2008). Educación física y mercado laboral. Competencias profesionales. Cultura, ciencia y deporte, 8, 123-131. Recuperado 
dehttp://ccd.ucam.edu/index.php/revista/article/view/187

Lledó, E., Martínez, G., \& Huertas, F. (2014). Perfil del entrenador de fútbol enla etapa escolar en escuelas de clubes deélitedela Comunitat Valencian. Cultura, ciencia y deporte, 9(25), 57-68. Recuperado de http://ccd.ucam.edu/index.php/revista/article/view/389/287

López, V. M., \& Gea, J. M. (2010). Innovación, discurso y racionalidad en educación física. Revisión y prospectiva. Revista Internacional de Medicinay Ciencias de la Actividad Física y del Deporte, 10(38), 245-270. Recuperado de http://cdeporte.rediris.es/revista/revista38/ artinnovacion154.htm

López, V. M., Monjas, R., \& Fraile, A. (Eds.). (2004). Los últimos diez años de la educación física escolar. Valladolid: Universidad de Valladolid.

Martínez, L. (2016). Las prácticas de los maestros especialistas de educación física desde la perspectiva del coordinador del programa: una aproximación narrativa (Tesis Doctoral inédita). Departamento de Expresión Musical, Plástica y Corporal de la Universidad de Valladolid, Palencia.

Mosston, M. (1978). La enseñanza de la educación física: del comando al descubrimiento. Buenos Aires: Paidós.

Mosston, M., \& Ashworth, S. (1993). La enseñanza de la educación física: la reforma de los estilos de enseñanza. Barcelona: Hispano Europea.

Pastor, J. L. (1997). El espacio profesional de la Educación Física en España: génesis y formación (1883-1961). Guadalajara: Servicio de Publicaciones de la Universidad de Alcalá.

Pastor, J. L. (2000). Definición y desarrollo del espacio profesional de la Educación Física en España (1961-1990). Guadalajara: Servicio de Publicaciones de la Universidad de Alcalá.

Pastor, J. L. (2003). La investigación en las ciencias de la actividad física y el deporte: : perspectiva histórica. Agora para la educación física y el deporte, 2, 39-50. Recuperado de http://www5.uva.es/agora/ revista/2/agora2_3_pastorpradillo.pdf

Pastor, J. L. (2004). ¿Q Quo vadis gimnástica? Aproximación histórica a la evolución de una profesión. Revista española de educación físicay deportes, 1, 15-38. Recuperado de http://www.consejo-colef.es/ revista-reefd/archivo-revista-reefd/4-revista-375/download.html

Pérez-Gutiérrez, M., \& Gutiérrez-García, C. (2012). Estudio bibliométrico sobre las monografías de artes marciales publicadas en España (1906-2006). Revista de Artes Marciales Asiáticas, 3(4), 22-33. doi:http://dx.doi.org/10.18002/rama.v3i4.387

Pérez-Gutiérrez, M., Gutiérrez-García, C., \& Álvarez, E. (2013). Repertorio bibliográfico anotado de monografías de artes marciales asiáticas publicadas en España. Revista de Artes Marciales Asiáticas, 8(1), 21-450. doi:http://dx.doi.org/10.18002/rama.v8i1.836

Piéron, M. (1988). Didáctica de las actividades físicas y deportivas. Madrid: Gymnos.

Piéron, M. (1999). Para una enseñanza eficaz de las actividades físicodeportivas. Barcelona: Inde.

Romero, C., Zagalaz, M. L., Romero, M. N., \& Martínez, E. J. (2011). Importancia de las competencias profesionales de los Maestros en Educación Física expresadas por los estudiantes. Retos: nuevas tendencias en educación física, deporte y recreación, 19, 62-68. Recuperado de http://recyt.fecyt.es/index.php/retos/article/view/ 34641

Sánchez, F. (1984). Bases para una didáctica de la educación física yel deporte. Madrid: Gymnos.

Sancho, J. M. (2001). Docencia e investigación en la universidad: una profesión, dos mundos. Educar, 28, 41-60. Recuperado de http:// www.raco.cat/index.php/Educar/article/download/20742/20582

Seners, P. (2001). La lección de educación física. Barcelona: Inde.

Sicilia, Á., \& Delgado, M. A. (2002). Educación física y estilos de enseñanza. Barcelona: Inde.

Sicilia, Á., \& Fernández-Balboa, J. M. (Eds.). (2005). La otra cara de la enseñanza. La educación física desde una perspectiva crítica. Barcelona: Inde.

Siedentop, D. (2008). Aprender a enseñar la educación física. Barcelona: Inde.

Ureña, F. (Ed.). (2010). La Educación Física en Secundaria basada en competencias (5 vols.). Barcelona: Inde.

Valdemoros, M. A. (2010). Los valores en el ocio físico-deportivo: Análisis y propuestas educativas. La Rioja: Universidad de La Rioja.

Valenciano, J. (2012). Las funciones sociales de la investigación a través de las revistas iberoamericanas de Educación Física. ¿Comprometidas con la transformación social? Estudios Pedagógicos, 38 ( ${ }^{\circ}$ especial 1), 293-309. Recuperado de http://dx.doi.org/10.4067/ S0718-07052012000400016

Valenciano, J., Devís-Devís, J., \& Villamón, M. (2008). Revistas científicas y evaluación de la actividad investigadora en las Ciencias de la Actividad Física y el Deporte. Apunts: Educación física y deportes, 94, 7-13. Recuperado de http://www.revista-apunts.com/es/ hemeroteca?article $=1275$

Valenciano, J., Devís-Devís, J., Villamón, M., \& Peiró, C. (2010). La colaboración científica en el campo de las Ciencias de la Actividad Física y el Deporte en España. Revista española de documentación científica,33(1), 90-105. doi:10.3989/redc.2010.1.726

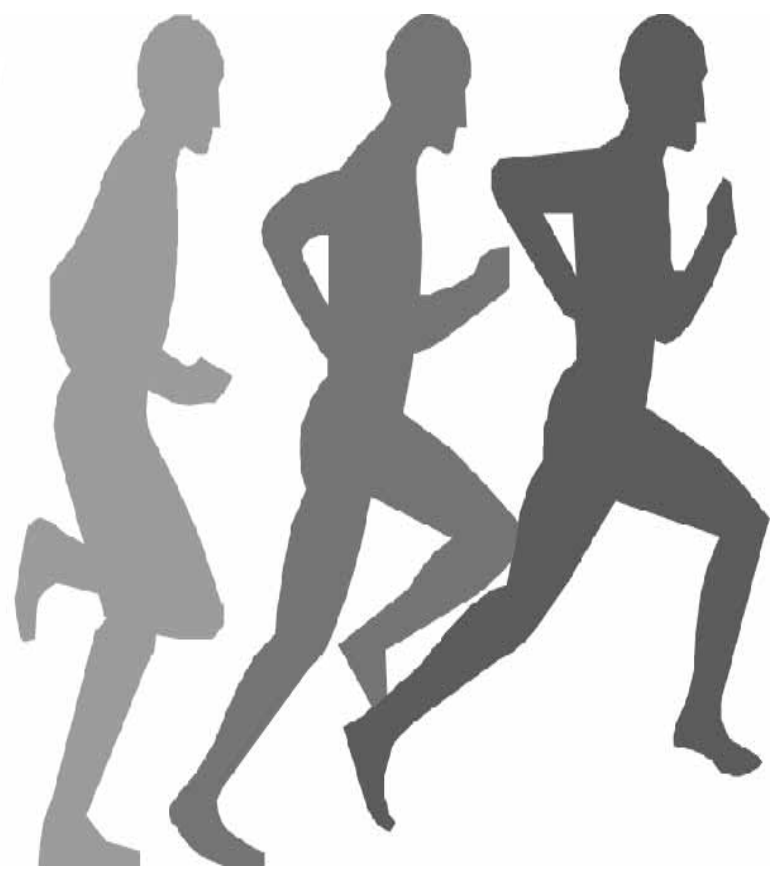

